\title{
Diferentes estratégias alimentares na larvicultura do acará-bandeira (Peterolophyllum scalare, Cichlidae)
}

\author{
Samuel Louzada PEREIRA*, Lucas Pedro GONÇALVES JUNIOR², Rafael Vieira de AZEVEDO ${ }^{1}$, Marcelo \\ Darós MATIELO², Paula del Caro SELVATICI², Ismael Rafane AMORIM ${ }^{3}$, Pedro Pierro MENDONÇA ${ }^{3}$ \\ ${ }_{1}^{*}$ Universidade Estadual do Norte Fluminense Darcy Ribeiro. Laboratório de Zootecnia. Campos dos Goytacazes, Rio de Janeiro, Brasil. \\ ${ }^{2}$ Universidade Federal do Espírito Santo. Alegre, Espírito Santo, Brasil. \\ ${ }^{3}$ Instituto Federal do Espírito Santo, Campus de Alegre. Laboratório de nutrição e produção de espécies ornamentais. Alegre, Espírito Santo, Brasil. \\ * Autor correspondente: samuell_Ip@hotmail.com.
}

\begin{abstract}
RESUMO
A larvicultura é uma das etapas mais críticas do desenvolvimento dos peixes e o seu sucesso está diretamente relacionado ao manejo alimentar, que pode proporcionar maiores sobrevivência e crescimento. Objetivou-se avaliar o tempo de transição alimentar e de fornecimento de meta-náuplios de Artemia spp. (MNA) na larvicultura do acará-bandeira. Dois experimentos foram conduzidos em delineamento inteiramente casualizado com cinco tratamentos e quatro repetiçóes. Em cada experimento foram utilizados 540 peixes distribuídos em 20 aquários com 2 L. No primeiro experimento, avaliaram-se os períodos de transição alimentar (MNA + ração) por 1, 2, 3, 4 e 5 dias. No segundo experimento, avaliou-se o período de fornecimento de MNA por 5, 10, 15, 20 e 25 dias. Foram avaliados: ganho de peso, taxas de crescimento e desenvolvimento específico, sobrevivência e uniformidade do lote (apenas no experimento para avaliar o tempo de fornecimento de MNA). Não houve efeito significativo dos diferentes períodos de transição alimentar sobre as variáveis de crescimento $(p>0,05)$, porém a sobrevivência foi maior $(p<0,05)$ nos tratamentos compostos por 3, 4 e 5 dias de alimentação conjunta. Em relação ao tempo de fornecimento de MNA, foram observados piores resultados $(p<0,05)$ quando o tempo de fornecimento do alimento vivo foi menor $(5$, 10 e 15 dias). Os animais que foram alimentados com MNA antes da transição alimentar, por mais tempo (20 e 25 dias), apresentaram os melhores resultados de crescimento $(p<0,05)$. Portanto, recomenda-se uma transiçáo alimentar de três dias e um fornecimento de MNA por 20 dias para realizar a substituição total do alimento vivo pela ração.
\end{abstract}

PALAVRAS-CHAVE: Alimentação, peixe ornamental, ração, sobrevivência.

\section{Different feeding strategies on larval rearing of angelfish (Peterolophyllum scalare, Cichlidae)}

\begin{abstract}
The hatchery is one of the most critical stages of the development of fish and their success is directly related to food handling that can provide greater survival and growth. The aim of this study was to evaluate the time of dietary transition and providing meta-nauplii of Artemia spp. (MNA) for the angelfish larvae. Two experiments were conducted in a completely randomized design with five treatments and four repetitions. On each experiment there were used 540 fishes, distributed in 20 tanks with 2 liters each. In the first experiment, there were evaluated the dietary transition periods (MNA + Diet) for 1, 2, 3, 4 and 5 days. In the second experiment, there were evaluated the supply of MNA for 5, 10, 15, 20 and 25 days. There were evaluated: weight gain, growth rate and specific development, survival and batch uniformity (only in the experiment to evaluate the time of fish feed with MNA). There was no significant effect of different periods of dietary transition on the growth variables $(p>0.05)$, but survival was higher $(p<0.05)$ in the treatments composed of 3,4 and 5 days of joint feed. In relation to the time of supply of MNA worse results were observed $(p<0.05)$ when the time of supply of live food was shorter $(5,10$ and 15 days). The animals were fed with MNA before feeding transition, for longer time (20 and 25) showed the best results for growth $(p<0.05)$. Therefore, a food transition of three days and a supply of MNA for 20 days is recommended to perform the total replacement of live food for feed.
\end{abstract}

KEYWORDS: Feeding, ornamental fish, feed, survival. 


\section{INTRODUÇÃO}

Dentre as diversas espécies de peixes ornamentais produzidas, destaca-se o acará-bandeira (Pterophyllum scalare, Lichtenstein, 1823, Cichlidae), por ser um peixe de grande comercializaçáo, exuberância e também um dos mais populares de águas tropicais (Chapman et al. 1997). Esta espécie é originária da Bacia Amazônica, amplamente distribuída, com ocorrência no Peru, Colômbia, Guianas e Brasil. $\mathrm{O}$ acará bandeira é um peixe pacifico, que nada em cardumes, principalmente quando jovem. No entanto, quando adultos, demonstram comportamento hierárquico dentro dos cardumes, o que faz com que alguns animais sejam subjugados por outros mais desenvolvidos. Além disso, peixes desta espécie possuem boa adaptação a diferentes ambientes, o que facilita sua comercialização (Ribeiro et al. 2007).

Devido à relevância da espécie, faz-se necessário o desenvolvimento de técnicas que melhorem as características produtivas na larvicultura desta espécie, uma vez que se trata da fase mais crítica de desenvolvimento do peixe. Segundo Hung et al. (1989), a maioria dos peixes náo aceita, na fase inicial, alimentos artificiais, e os que o fazem, apresentam crescimento reduzido, que resulta em problemas no desenvolvimento subsequente. No início da alimentação exógena, as pós-larvas apresentam elevadas exigências nutricionais, devido a altas taxas de crescimento. Desta forma, um dos fatores críticos na larvicultura é a alimentaçáo, onde deve ser fornecido ao animal alimento que supra os requerimentos nutricionais da espécie.

A substituição precoce do alimento vivo, ou seja, transição alimentar, pode diminuir os custos de produção (Jomori et al. 2008). No entanto, apesar dos avanços obtidos nas pesquisas, a maioria dos trabalhos relata que, embora seja possível, ainda é inviável economicamente a utilização de alimento inerte como primeiro alimento exógeno no início da larvicultura (Tesser $e t$ al. 2005; Menossi et al. 2012). A substituição muito precoce do alimento vivo pelo inerte tem levado a uma redução no crescimento e na sobrevivência das pós-larvas (Jomori et al. 2008; Puello-cruz et al. 2010; Jelkic et al. 2012) comparandose ao crescimento observado com maior tempo de oferta do alimento vivo (Lombardi e Gomes 2008). Assim, com o presente estudo, objetivou-se avaliar o tempo de transição alimentar e de fornecimento de meta-náuplios de Artemia spp. na larvicultura do acará-bandeira.

\section{MATERIAL E MÉTODOS}

Foram realizados dois experimentos no Instituto Federal do Espírito Santo - Campus de Alegre - no Laboratório de Nutrição e Produção de Espécies Ornamentais.

Um total de 1.080 pós-larvas de acará-bandeira (Pterophyllum scalare, Lichtenstein, 1823, Cichlidae) foi utilizado para a realizaçáo dos experimentos. No início, 140 pós-larvas, em cada experimento, foram separadas para a realização das medições iniciais de peso $(0,001 \mathrm{~g})$ com balança analítica (Balança Analítica Adventurer Pro 260g, Ohaus Corporation, New Jersey, USA), comprimento total $(0,01$ $\mathrm{mm}$ ) com paquímetro (Paquímetro Universal, Digimess, São Paulo, SP, Brasil) e, posteriormente, descartadas.

\section{Experimento 1}

O primeiro experimento foi realizado para determinar o período de transição alimentar, em delineamento inteiramente casualizado, com cinco tratamentos e quatro repetiçôes. Um total de 400 pós-larvas $(1,14 \pm 0,06 \mathrm{mg}$ e 4,26 $\pm 0,48 \mathrm{~mm})$ foi distribuído em 20 aquários com volume útil de $2 \mathrm{~L}$, em sistema de banho termostatizado.

Em todos os tratamentos, as pós-larvas receberam inicialmente 10 dias de alimentação composta exclusivamente por meta-náuplios de Artemia spp. No décimo primeiro dia, iniciou-se a alimentação conjunta (transiçáo alimentar) com meta-náuplios de Artemia spp. e ração comercial nos períodos de 1, 2, 3, 4 ou 5 dias, sendo estes períodos os tratamentos 1 , 2, 3, 4 e 5, respectivamente. Após cada período de transiçáo alimentar, as pós-larvas receberam apenas ração (Tabela 1).

A alimentação foi realizada três vezes ao dia $(7 \mathrm{~h} 00,11 \mathrm{~h} 30$ e 16h00). Para a obtenção dos meta-náuplios de Artemia spp., utilizou-se três gramas de cistos em um recipiente plástico transparente, com volume útil de $2 \mathrm{~L}$ provido de aeração e iluminação constantes. A salinidade da água, durante a incubação dos cistos, foi mantida em 30 ppmil. Após 24 horas, realizou-se a filtragem em rede organza e os metanáuplios de Artemia spp. foram fornecidos às pós-larvas. Para aferição da quantidade de meta-náuplios de artêmia ofertados nas unidades experimentais, foi realizado, antes de cada alimentaçáo, a contagem destes. Para isso, os metanáuplios de Artemia spp. foram concentrados em peneiras e estocados em Becker graduado, contendo 2 L de água salinizada, sob aeração, retirada uma amostra de $1 \mathrm{~mL}$ e diluída 50 vezes. Desse volume foram retiradas sub-amostras de $1 \mathrm{~mL}$ e quantificados os meta-náuplios em placa de Petri sob iluminação.

A quantidade de alimento vivo ofertada para cada pós-larva foi de 75 meta-náuplios de Artemia spp. pós-larva ${ }^{-1}$ dia $^{-1}$, até o décimo dia, ajustando-se para 100 meta-náuplios de Artemia spp. pós-larva ${ }^{-1}$ dia $^{-1}$ até o término da transição alimentar. A raçáo comercial era composta de $55 \%$ de proteína bruta, $7 \%$ de extrato etéreo, 2,8\% de matéria fibrosa, $1,70 \%$ de matéria mineral.

Após 20 minutos de cada alimentação foi realizada sifonagem das unidades experimentais, renovando-se aproximadamente $50 \%$ do seu volume. Os parâmetros de qualidade de água oxigênio dissolvido, temperatura, $\mathrm{pH}$ e condutividade elétrica foram mensurados diariamente 
Tabela 1.Representação do período de fornecimento dos diferentes alimentos utilizados, do início ao término do período experimental, em dias, e a composição da dieta durante o período de transição alimentar.

\begin{tabular}{|c|c|c|c|c|}
\hline \multirow{2}{*}{ Tratamento } & \multirow{2}{*}{ Período(dias) } & \multirow{2}{*}{$\begin{array}{l}\text { Estratégia alimentar utilizada durante o período } \\
\text { experimental }\end{array}$} & \multicolumn{2}{|c|}{ Porcentagem da dieta utilizada (\%) } \\
\hline & & & Artemia spp. & Ração \\
\hline \multirow{3}{*}{1} & $1-9$ & Somente Alimento vivo & 100 & 0 \\
\hline & $10-11$ & Alimento vivo + ração & 50 & 50 \\
\hline & $12-30$ & Somente ração & 0 & 100 \\
\hline \multirow{3}{*}{2} & $1-9$ & Somente Alimento vivo & 100 & 0 \\
\hline & $10-12$ & Alimento vivo + ração & 50 & 50 \\
\hline & $13-30$ & Somente ração & 0 & 100 \\
\hline \multirow{3}{*}{3} & $1-9$ & Somente Alimento vivo & 100 & 0 \\
\hline & $10-13$ & Alimento vivo + ração & 50 & 50 \\
\hline & $14-30$ & Somente ração & 0 & 100 \\
\hline \multirow{3}{*}{4} & $1-9$ & Somente Alimento vivo & 100 & 0 \\
\hline & $10-14$ & Alimento vivo + ração & 50 & 50 \\
\hline & $15-30$ & Somente ração & 0 & 100 \\
\hline \multirow{3}{*}{5} & $1-9$ & Somente Alimento vivo & 100 & 0 \\
\hline & $10-15$ & Alimento vivo + ração & 50 & 50 \\
\hline & $16-30$ & Somente ração & 0 & 100 \\
\hline
\end{tabular}

com a utilização de, respectivamente, oxímetro digital com termômetro (DO-5519, Lutron Electronic Enterprise Co., LTD, Taipei, Taiwan), peagâmetro portátil (PH100 Phtek, Sdorf Laboratorial, São Paulo, SP, Brasil) e condutivímetro (Lutron CD 4301, Lutron Electronic Enterprise Co., LTD, Taipei, Taiwan).

Ao final de 30 dias foram realizadas as mediçóes finais de todas as pós-larvas sobreviventes, obtendo-se o peso $(0,001$ g) e comprimento $(0,01 \mathrm{~mm})$ e calculados: ganho de peso, através da diferença entre os pesos médios final e inicial; taxa de crescimento específico utilizando a equação [(ln peso médio final - ln peso médio inicial) / período experimental] $\mathrm{x}$ 100; taxa de desenvolvimento específico utilizando a equação [(ln comprimento total médio final - ln comprimento total médio inicial) / período experimental] x 100; e sobrevivência (número de pós-larvas final / número de pós-larvas inicial) $\mathrm{x} 100$.

\section{Experimento 2}

O segundo experimento foi realizado para determinar o período de fornecimento de meta-náuplios de Artemia spp., em um delineamento inteiramente casualizado, com cinco tratamentos e quatro repetiçóes. Um total de 400 pós-larvas $(1,13 \pm 0,02 \mathrm{mg}$ e 4,27 $\pm 0,33 \mathrm{~mm})$ foi distribuído em 20 aquários com volume útil de $2 \mathrm{~L}$, em sistema de banho termostatizado.

Inicialmente, as pós-larvas receberam meta-náuplios de Artemia spp. em diferentes períodos, a saber: 5, 10, 15,
20 e 25 dias, sendo estes períodos os tratamentos 1, 2, 3, 4 e 5, respectivamente. Após os respectivos períodos de fornecimento de meta-náuplios de Artemia spp., iniciou-se a transição alimentar de três dias, baseando-se no melhor resultado obtido no experimento 1 . Após esse período, os animais foram alimentados somente com ração experimental até o término do período experimental (Tabela 2).

Os manejos com alimentação e qualidade de água foram realizados conforme descrito no experimento 1 , diferenciando-se apenas no número de meta-náuplios de Artemia spp. pós-larva ${ }^{-1}$ dia $^{-1}$ (Tabela 3).

Ao final de 30 dias foram realizadas as mediçóes finais de todas as pós-larvas sobreviventes e obtidos os parâmetros para avaliação descritos no experimento 1 . Além destes, para avaliar a uniformidade do lote (U\%), utilizou-se a equação $(\mathrm{N} \pm 10 \% \times 100) / \mathrm{Nt}$; em que, $\mathrm{N} \pm 10 \%$, é o número de animais com comprimento padrão total $\pm 10 \%$ em torno da média da unidade experimental e $\mathrm{Nt}$ é o número total de peixes em cada unidade experimental.

Para a análise estatística dos dados obtidos nos experimentos, os valores em porcentagem passaram por transformação arco seno da raiz quadrada do valor percentual. Após a verificação de normalidade e homocedasticidade, os resultados foram submetidos à análise de variância a 5\% de significância. Quando "F" foi significativo, utilizou-se teste Tukey a 5\% através do programa do Statistical Analysis System 9.0. 
Tabela 2. Representação dos tratamentos utilizados no experimento de tempo de fornecimento de alimento vivo e porcentagem de alimento fornecido durante o período experimental.

\begin{tabular}{|c|c|c|c|c|}
\hline \multirow{2}{*}{ Tratamento } & \multirow{2}{*}{$\begin{array}{l}\text { Período } \\
\text { (dias) }\end{array}$} & \multirow{2}{*}{$\begin{array}{l}\text { Estratégia alimentar utilizada durante o período } \\
\text { experimental }\end{array}$} & \multicolumn{2}{|c|}{ Porcentagem da dieta utilizada (\%) } \\
\hline & & & Artemia spp. & Ração \\
\hline \multirow{3}{*}{1} & $1-5$ & Somente Alimento vivo & 100 & 0 \\
\hline & $6-8$ & Alimento vivo + ração & 50 & 50 \\
\hline & $9-30$ & Somente ração & 0 & 100 \\
\hline \multirow{3}{*}{2} & $1-10$ & Somente Alimento vivo & 100 & 0 \\
\hline & $11-13$ & Alimento vivo + ração & 50 & 50 \\
\hline & $14-30$ & Somente ração & 0 & 100 \\
\hline \multirow{3}{*}{3} & $1-15$ & Somente Alimento vivo & 100 & 0 \\
\hline & $15-18$ & Alimento vivo + ração & 50 & 50 \\
\hline & $19-30$ & Somente ração & 0 & 100 \\
\hline \multirow{3}{*}{4} & $1-20$ & Somente Alimento vivo & 100 & 0 \\
\hline & $21-23$ & Alimento vivo + ração & 50 & 50 \\
\hline & $24-30$ & Somente ração & 0 & 100 \\
\hline \multirow{3}{*}{5} & $1-25$ & Somente Alimento vivo & 100 & 0 \\
\hline & $26-28$ & Alimento vivo + ração & 50 & 50 \\
\hline & $29-30$ & Somente ração & 0 & 100 \\
\hline
\end{tabular}

Tabela 3. Manejo alimentar commeta-náuplios deArtemiaspp.realizado no experimento detempo de fornecimento de alimento vivo

\begin{tabular}{cccccc}
\hline \multirow{2}{*}{$\begin{array}{c}\text { Período } \\
\text { (dias) }\end{array}$} & \multicolumn{5}{c}{ Número de meta-náuplios de Artemia spp. pós-larva-1 dia $^{-1}$} \\
\cline { 2 - 6 } & 1 & 2 & 3 & 4 & 5 \\
\cline { 2 - 6 } & 75 & 75 & 75 & 75 & 75 \\
\hline $1-8$ & - & 100 & 100 & 100 & 100 \\
\hline $9-13$ & - & - & 100 & 100 & 100 \\
\hline $9-18$ & - & - & - & 150 & 150 \\
\hline $19-23$ & - & - & - & - & 200 \\
\hline $24-28$ & & & &
\end{tabular}

\section{RESULTADOS}

\section{Experimento 1}

Os parâmetros físicos e químicos da água foram semelhantes $(p>0,05)$ entre os tratamentos. Os valores de oxigênio dissolvido, $\mathrm{pH}$, condutividade elétrica e temperatura foram, respectivamente, $5,38 \pm 0,32 \mathrm{mg} \mathrm{L}^{-1}, 6,98 \pm 0,10,0,59$ $\pm 0,04 \mu \mathrm{S} . \mathrm{cm}$ e $27,35 \pm 0,06^{\circ} \mathrm{C}$.

Ao final do período experimental, observou-se que apenas a sobrevivência apresentou diferença estatística, com os melhores resultados para esse parâmetro a partir do terceiro dia de transição alimentar (Tabela 4). Os valores para sobrevivência foram maiores $(p<0,05)$ nos tratamentos com três, quatro e cinco dias de transição alimentar. Em relação ao peso final e ganho de peso, não foram observadas diferenças estatísticas $(p>0,05)$.

\section{Experimento 2}

Os parâmetros físicos e químicos da água foram semelhantes $(p>0,05)$ entre os tratamentos. Os valores de oxigênio dissolvido, $\mathrm{pH}$, condutividade elétrica e temperatura foram, respectivamente, $5,25 \pm 0,40 \mathrm{mg} \mathrm{L}^{-1}, 7,03 \pm 0,05,0,60$ $\pm 0,01 \mu \mathrm{S} . \mathrm{cm}$ e $27,29 \pm 0,15^{\circ} \mathrm{C}$.

Para o peso final, foram observados piores $(p<0,05)$ resultados quando o tempo de fornecimento do alimento vivo foi menor (Tabela 5). Os animais que foram alimentados com meta-náuplios de Artemia spp. por período maior, como observado em 20 e 25 dias, apresentaram os melhores resultados para comprimento total, ganho de peso, taxa de crescimento específico e taxa de desenvolvimento específico $(p<0,05)$.

Para sobrevivência, observou-se os melhores resultados $(p<0,05)$ nos tratamentos com 15, 20 e 25 dias de fornecimento de alimento vivo, enquanto para a uniformidade do lote, calculada com base dos resultados obtidos de comprimento padrão dos animais, os maiores resultados $(p<0,05)$ foram observados com menores períodos de fornecimento de metanáuplios de Artemia spp. (Figura 1).

\section{DISCUSSÃO}

Nos dois experimentos, os parâmetros físicos e químicos da água permaneceram dentro da faixa indicada para o adequado desenvolvimento do acará-bandeira, conforme recomendado por Pérez et al. (2003) e Takahashi et al. (2010). Segundo esses autores, o acará-bandeira tolera ampla faixa para a maioria dos 
Tabela 4.Valores médios \pm desvio padrão das variáveis peso final (PF), comprimento total (CT), ganho de peso (GP), taxa de crescimento específico (TCE), taxa de desenvolvimento específico (TDE) e sobrevivência (SOB) das larvas de acará-bandeira de acordo com o período de transição alimentar.

\begin{tabular}{cccccc}
\hline \multirow{2}{*}{ Variável } & \multicolumn{5}{c}{ Tratamento (dias de transição alimentar) } \\
\cline { 2 - 6 } & 1 & 2 & 3 & 4 & 5 \\
\hline PF (mg) & $41,07 \pm 2,71$ & $37,27 \pm 5,12$ & $42,19 \pm 4,88$ & $43,72 \pm 1,64$ & $44,70 \pm 3,55$ \\
CT (cm) & $1,47 \pm 0,28$ & $1,45 \pm 0,22$ & $1,54 \pm 0,15$ & $1,57 \pm 0,31$ & $1,56 \pm 0,11$ \\
GP (mg) & $39,93 \pm 2,77$ & $36,12 \pm 4,21$ & $41,04 \pm 4,88$ & $42,58 \pm 5,11$ & $43,56 \pm 5,41$ \\
TCE (\%dia $\left.{ }^{-1}\right)$ & $19,37 \pm 1,18$ & $19,29 \pm 2,33$ & $19,41 \pm 2,26$ & $19,44 \pm 1,69$ & $19,46 \pm 3,00$ \\
TDE $\left(\%\right.$ dia $\left.^{-1}\right)$ & $3,22 \pm 0,18$ & $3,21 \pm 0,61$ & $3,27 \pm 0,12$ & $3,29 \pm 0,20$ & $3,28 \pm 0,11$ \\
SOB (\%) & $90,00 \pm 7,07^{\mathrm{B}}$ & $90,00 \pm 12,25^{\mathrm{B}}$ & $95,00 \pm 0,00^{\mathrm{A}}$ & $95,00 \pm 3,54^{\mathrm{A}}$ & $95,00 \pm 5,00^{\mathrm{A}}$ \\
\hline
\end{tabular}

Médias seguidas por letras diferentes, nas linhas, diferem entre si $(P<0.05)$ pelo teste de Tukey

Tabela 5.Valores médios \pm desvio padrão das variáveis peso final (PF), comprimento total (CT), ganho de peso (GP), taxa de crescimento específico (TCE) e taxa de desenvolvimento específico (TDE) das larvas de acará-bandeira de acordo com o tempo de fornecimento de alimento vivo

\begin{tabular}{llllll}
\hline \multirow{2}{*}{ Variável } & \multicolumn{4}{l}{ Tratamento (dias de alimento vivo) } & \multicolumn{4}{l}{20} & 25 \\
\cline { 2 - 6 } & 5 & 10 & 15 & $52,79 \pm 3,48^{\mathrm{A}}$ & $62,7 \pm 4,77^{\mathrm{A}}$ \\
\hline PF $(\mathrm{mg})$ & $34,80 \pm 3,88^{\mathrm{C}}$ & $36,48 \pm 5,61^{\mathrm{BC}}$ & $49,03 \pm 5,11^{\mathrm{AB}}$ & $1,69 \pm 0,14^{\mathrm{AB}}$ & $1,79 \pm 0,11^{\mathrm{A}}$ \\
CT $(\mathrm{cm})$ & $1,47 \pm 0,38^{\mathrm{C}}$ & $1,49 \pm 0,22^{\mathrm{C}}$ & $1,62 \pm 0,31^{\mathrm{BC}}$ & $51,65 \pm 5,39^{\mathrm{A}}$ & $61,60 \pm 4,44^{\mathrm{A}}$ \\
GP $(\mathrm{mg})$ & $33,65 \pm 5,38^{\mathrm{C}}$ & $35,34 \pm 6,33^{\mathrm{BC}}$ & $47,88 \pm 3,33^{\mathrm{AB}}$ & $12,71 \pm 2,01^{\mathrm{A}}$ & $13,34 \pm 1,99^{\mathrm{A}}$ \\
TCE $\left(\%\right.$ dia $\left.^{-1}\right)$ & $11,38 \pm 1,29^{\mathrm{B}}$ & $11,54 \pm 2,22^{\mathrm{B}}$ & $12,52 \pm 1,93^{\mathrm{A}}$ & $4,59 \pm 0,55^{\mathrm{AB}}$ & $4,77 \pm 0,29^{\mathrm{A}}$ \\
\hline
\end{tabular}

Médias seguidas por letras diferentes, nas linhas, diferem entre si $(P<0.05)$ pelo teste de Tukey

parâmetros de qualidade da água, desde que essas variaçôes ocorram gradativamente.

\section{Experimento 1}

Embora não tenha sido observado efeito significativo do período de transição alimentar sobre o crescimento, os valores de sobrevivência foram superiores a partir de três dias de transição alimentar, indicando que esse período foi suficiente para realizar a troca do alimento vivo pelo alimento inerte durante a larvicultura do acará-bandeira. As pós-larvas de acará-bandeira alimentadas com alimento vivo até o décimo dia de vida (três dias de transiçáo alimentar), provavelmente já apresentavam o sistema digestório funcional, o que resultou em melhor aproveitamento do alimento inerte após a substituiçáo do alimento vivo.

Geralmente, as larvas de peixes altriciais apresentam dificuldades relacionadas a aceitaçáo e utilizaçáo do alimento inerte, recomendando-se período maior para retirar totalmente o alimento vivo e fornecer apenas o alimento inerte. Esse fato náo foi verificado para o acará-bandeira, diferindo do encontrado para outras espécies, como nos estudos realizados por Rocha et al. (2008) com o linguado (Paralichthys orbignyanus) e Callan et al. (2003) com o bacalhau (Gadus morhua). Segundo esses autores, a transição alimentar de forma gradativa favorece a aceitaçáo do alimento inerte, acelera o processo de substituiçáo do alimento vivo e facilita o processo de digestão e assimilação de nutrientes.
No entanto, ressalta-se a importância do alimento vivo nos primeiros dias de vida. Menossi et al. (2012) compararam os efeitos de diferentes dietas e da transição alimentar sobre o desempenho zootécnico e a morfologia do trato digestivo de pós-larvas de pacu. Os melhores resultados para desempenho e sobrevivência, assim como mais acelerada organogênese do sistema digestório foram verificados entre os animais préalimentados com alimento vivo. Já os animais submetidos aos tratamentos que utilizaram apenas dieta inerte, apresentaram os piores resultados de desempenho. Callan et al. (2003)

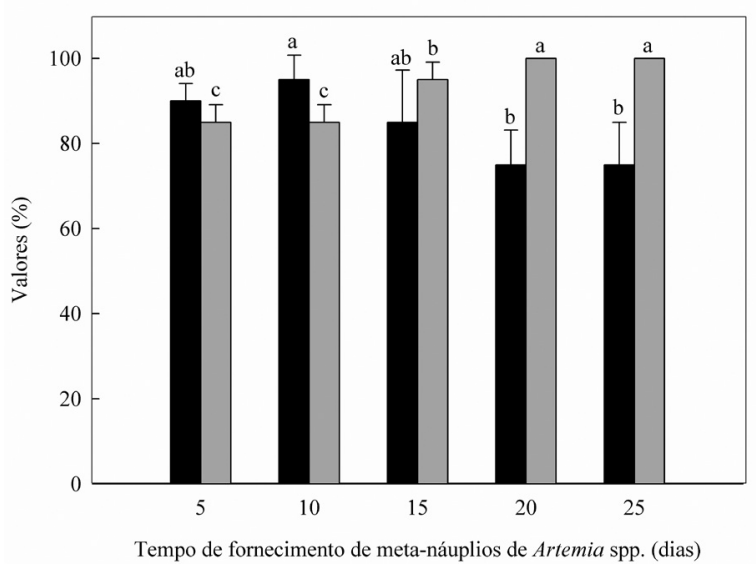

Figura 1. Valores médios \pm desvio padrão de sobrevivência e uniformidade do lote no experimento de tempo de fornecimento de alimento vivo. Coloração escura: Uniformidade do lote e Coloração clara: Sobrevivência. 
verificaram efeito negativo da substituição precoce do alimento vivo pelo inerte na larvicultura do bacalhau (Gadus morhua). Quando o alimento vivo correspondeu a acima de $25 \%$ da dieta, não houve efeito sobre o comprimento, peso, taxa crescimento especifico e sobrevivência.

\section{Experimento 2}

Neste estudo, verificou-se a influência do tempo de fornecimento de alimento vivo sobre o crescimento, sobrevivência e uniformidade do lote de pós-larvas de acarábandeira. Luna-Figueroa et al. (2010) realizaram estudo com pós-larvas de acará-bandeira e, nos tratamentos em que os animais receberam alimento vivo, foi possível notar melhores resultados de crescimento.

Alguns autores salientaram que o meta-náuplio de Artemia spp. é indispensável durante estudos com fases iniciais de peixes altriciais e influencia diretamente na ingestáo, por meio de estímulos químicos, quando a liberação de aminoácidos livres ativa os receptores da pós-larva, estimulando o apetite e orientando-as para as presas (Tesser e Portella 2006). Além disso, a utilização da Artemia spp., segundo Kurokawa et al. (1996), auxiliou na digestão e absorção de raçôes microencapsuladas durante a transição alimentar, mediante a influência direta da composição bioquímica do meta-náuplio no processo de digestão e absorção dos nutrientes pelas póslarvas.

Yufera et al. (1996) avaliaram desenvolvimento das pós-larvas de Sparus aurata alimentadas com dietas microencapsuladas como a única fonte de alimento desde o início da alimentação. Estas pós-larvas apresentaram forte degeneração do epitélio intestinal, fígado e pâncreas e morreram dentro de dois a três dias após o início da alimentação. As pós-larvas criadas com uma dieta mista, composta da raçấo inerte e uma pequena quantidade de rotíferos $\left(0,5\right.$ rotíferos $\left.\mathrm{mL}^{-1}\right)$, mostraram desenvolvimento normal do epitélio intestinal. Apesar de pós-larvas submetidas a esses tratamentos apresentarem valores de desempenho satisfatórios, a sobrevivência foi reduzida à metade do obtido para os animais alimentados apenas com rotífero.

Quanto a sobrevivência, Diemer et al. (2012) encontraram resultados inferiores em pós-larvas que foram submetidos a troca abrupta de alimento vivo por inerte. Entretanto, a ausência de período de transição alimentar pode ter influenciado na reduçấo desse parâmetro. Diemer et al. (2010), em estudo com pós-larvas de mandi pintado, observou melhores resultados para os tratamentos com Artemia spp. e transição alimentar e piores resultados com pós-larvas alimentadas apenas com alimento inerte.

É possível identificar que a ausência do alimento vivo, sua substituição abrupta ou em períodos muito curtos, podem resultar na redução da sobrevivência durante a larvicultura
(Lombardi e Gomes 2008; Fosse et al. 2013), enquanto a utilização de alimentos vivos possibilitam melhores resultados de desempenho e sobrevivência (Luz e Zaniboni Filho 2001; Prieto et al. 2006; Rocha et al. 2008).

No presente estudo, observou-se que quanto maior o tempo de fornecimento de meta-náuplios de Artemia spp., menor a uniformidade do lote e maior a sobrevivência. Esses resultados podem estar relacionados ao fato de, dentre as pós-larvas alimentadas com maior tempo de metanáuplios de Artemia spp., algumas podem ter apresentado maior agilidade na captura e utilização do alimento vivo, e, consequentemente, apresentaram maior crescimento que outras, reduzindo a uniformidade do lote. Por outro lado, nos tratamentos onde foram fornecidos menores tempos de meta-náuplios de Artemia spp., as pós-larvas menores não se adaptaram ao alimento inerte, o que resultou na morte destas e, consequentemente, aumento na uniformidade das póslarvas remanescentes. Ribeiro et al. (2010) não encontraram diferença significativa na uniformidade do lote de pós-larvas de acará-bandeira em policultivo com camarão-da-Amazônia e destacaram o comportamento pacífico dos peixes em formas jovens. Os maiores valores para uniformidade do lote podem estar atribuídos a características da própria espécie, como a sociabilidade e o comportamento de viver em cardumes quando jovens (Gómez-Laplaza, 2006). Portanto, as interaçôes negativas do territorialismo não são intensas o suficiente para justificar redução desse parâmetro.

\section{CONCLUSÃO}

O fornecimento de alimento vivo é indispensável durante a fase inicial de vida do acará-bandeira. No presente estudo, as pós-larvas de acará-bandeira apresentaram os melhores resultados quando alimentadas por meta-náuplios de Artemia spp. por um maior período. Recomenda-se período de transição alimentar de três dias e tempo mínimo de fornecimento de meta-náuplios de Artemia spp. de 20 dias para realizar a substituição total do alimento vivo pela raçáo na larvicultura do acará-bandeira.

\section{AGRADECIMENTOS}

Os autores agradecem ao Instituto Federal do Espírito Santo - Campus de Alegre, pelo fornecimento dos animais e estrutura física utilizada para realizaçáo do estudo.

\section{BIBLIOGRAFIA CITADA}

Callan, C.; Jordaan, A.; Kling, L.J. 2003. Reducing Artemia use in the culture of Atlantic cod (Gadus morhua). Aquaculture,

219:585-595 
Chapman, F.A.; Fitz-Coy, S.A.; Thunberg, E.M.; Adams, C.M. 1997. United States of America trade in ornamental fish. Journal of the World Aquaculture Society, 28:1-10.

Diemer, O.; Neu, D.H.; Sary, C.; Feiden, A.; Boscolo, W.R.; Signor, A.A. 2010. Manejo alimentar na larvicultura do mandi-pintado Pimelodus britskii. Revista Brasileira de Saúde e Produção Animal, 11:903-908.

Diemer, O.; Neu, D.H.; Sary, C.; Finkler, J.K.; Boscolo, W.R.; Feiden, A. 2012. Artemia sp. na alimentaçáo de larvas de jundiá (Rhamdia quelen). Ciência Animal Brasileira, 12:175-179.

Fosse, P.J.; Mattos, D.C.; Cardoso, L.D. Motta, J.H.S.; Jasper, A.P.S.; Radael, M.C.; Andrade, D.R.; Vidal-Júnior, M.V. 2013. Estratégia de co-alimentação na sobrevivência e no crescimento de larvas de Betta splendens durante a transiçáo alimentar. Arquivo Brasileiro de Medicina Veterinária e Zootecnia, 65:1801-1807.

Gómez-Laplaza, L.M. 2006. Shoal choice in juvenile angelfish (Pterophyllum scalare): effects of social status and activity. Ethology Ecology and Evolution, 18:261-273.

Hung, S.S.O.; Lutes, P.B.; Conte, F.S. Storebakken, T. 1989. Growth and feed efficiency of white sturgeon (Acipenser transmontanus) sub-yearlings at different feeding rates. Aquaculture, 80: 147-153.

Jelkić, D.; Opačak, A.; Stević, I.; Ozimec, S.; Jug-Dujakovic, J.; Safner, R. 2012. Rearing carp larvae (Cyprinus carpio) in closed recirculatory system (ras). Croatian Journal of Fisheries, 70:9-17.

Jomori, R.K.; Ducatti, C.; Carneiro, D.J.; Portella, M.C. 2008. Stable carbon $(\delta 13 \mathrm{C})$ and nitrogen $(\delta 15 \mathrm{~N})$ isotopes as natural indicators of live and dry food in Piaractus mesopotamicus (Holmberg, 1887) larval tissue. Aquaculture Research, 39:370381.

Kurokawa, T.; Shiraishi, M.; Suzuki, T. 1996. Quantification of exogenous protease derived from zooplankton in the intestine of Japanese sardine (Sardinops melanotictus) larvae. Aquaculture, 161:491-499.

Lombardi, D.C.; Gomes, L.C. 2008. Substituição de alimento vivo por alimento inerte na larvicultura intensiva do tambacu (Colossoma macropomum X Piaractus mesopotamicus). Acta Scientiarum. Animal Science, 30:467-472.

Luna-Figueroa, J.; Vargas, Z.T.J.; Figueroa, T.J. 2010. Alimento vivo como alternativa en la dieta de larvas y juveniles de Pterophyllum scalare (Lichtenstein, 1823). Avances en Investigación Agropecuaria, 14:63-72.

Luz, R.K.; Zaniboni-Filho, E. 2001. Utilização de diferentes dietas na primeira alimentação do mandi-amarelo (Pimelodus maculatus, Lacépède). Acta Scientiarum, 23:483-489.

Menossi, O.C.C.; Takata, R.; Sánchez-Amaya, M.I.; Freitas, T.M.; Yúfera, M.; Portella, M.C. 2012. Crescimento e estruturas do sistema digestório de larvas de pacu alimentadas com dieta microencapsulada produzida experimentalmente. Revista Brasileira de Zootecnia, 41:1-10.

Pérez, E.; Diaz, F.; Espina, S. 2003.Thermoregulatory behavior and critical thermal limits of angel fish Pterophyllum scalare (Lichtenstein) (Pisces: Cichlidae). Journal of Thermal Biology, 28:531-537

Prieto, M.J.; Logato, P.V.R.; Moraes, G.F.; Okamura, D.; Araújo, F.G. 2006. Types of preys on growth and survival of pacu (Piaractus mesopotamicus) post-larvae. Ciência e Agrotecnologia, 30:1002-1007.

Puello-Cruz, A.; Velasco-Blanco, G.; Martínez-Rodríguez, I.E.; Felix-Ramos, E.; Voltolina, D. 2010. Growth and survival of siamese fighting fish, Betta Splendens, larvae at low salinity and with different diets. Journal of the World Aquaculture Society, 41:823-828

Ribeiro, F.A.S.; Jorge, P.H.; Fernandes, J.B.K.; Sakomura, N.K. 2010. Densidade de estocagem para produçáo de acará-bandeira em viveiros escavados em policultivo com camarão-da-amazônia. Revista Caatinga, 23:129-134.

Ribeiro, F.A.S.; Rodrigues, L.A.; Fernandes, J.B.K. 2007. Desempenho de juvenis de acará-bandeira (Pterophyllum scalare) com diferentes níveis de proteína bruta na dieta. Boletim do Instituto de Pesca, 33: 195-203.

Rocha, A.F.; Carvalho, C.V.A.; Sampaio, L.A. 2008. Produção de juvenis do linguado Paralichthys orbignyanus: efeito da duração do período de coalimentação durante o desmame. Ciência Rural, 38:2334-2338.

Takahashi, L.S.; Silva, T.V.; Fernandes, J.B.K. 2010. Efeito do tipo de alimento no desempenho produtivo de juvenis de acará-bandeira (Pterophyllum scalare). Boletim do Instituto de Pesca, 36:1-8.

Tesser, M.B., Carneiro D.J.; Portella M.C. 2005. Cofeeding of pacu (Piaractus mesopotamicus, Holmberg, 1887) larvae with Artemia nauplii and microencapsulated. Journal of Applied Aquaculture, 17:47-59.

Tesser, M.B.; Portella, M.C. 2006. Ingestão de raçáo e comportamento de larvas de pacu em resposta a estímulos químicos e visuais. Revista Brasileira de Zootecnia, 35:18871892.

Yúfera, M.; Sarasquete, M.C.; Fernández-Díaz, C.1996. Testing protein-walled microcapsules for the rearing of firstfeeding gilthead sea bream (Sparus aurata L.) larvae. Marine Freshwater Research. 47:211-216.

Recebido em 10/02/2015

Aceito em 22/06/2015 
